\title{
VECTOR-VALUED STOCHASTIC PROCESSES. V. OPTIONAL AND PREDICTABLE VARIATION OF STOCHASTIC MEASURES AND STOCHASTIC PROCESSES
}

\author{
NICOLAE DINCULEANU
}

(Communicated by William D. Sudderth)

\begin{abstract}
Let $\mu$ be a stochastic measure, with values in a Banach space $E$, with finite variation $|\mu|$. If $\mu$ is optional (resp. predictable), then $|\mu|$ is also optional (resp. predictable) provided $E$ is separable, or the dual of a separable space, or has the Radon-Nikodym property.

Let $A$ be a right continuous stochastic process with values in $E$, with finite variation $|A|$. If $A$ is measurable (resp. optional, predictable), then $|A|$, the continuous part $|A|^{c}$ and the discrete part $|A|^{d}$ have the same property.
\end{abstract}

1. Introduction. let $(\Omega, \mathcal{F}, P)$ be a probability measure space and $\left(\mathcal{F}_{t}\right)_{t \geq 0}$ a filtration satisfying the usual conditions. Denote $\mathcal{M}=B\left(R_{+}\right) \times \mathcal{F}$ and let $\mathcal{O}$ and $P$ be, respectively, the optional and the predictable $\sigma$-field. Let $E$ be a Banach space. Let $\mu: \mathcal{M} \rightarrow E$ be a stochastic measure with finite variation $|\mu|$. If $\mu$ is optional or predictable, is it true that its variation $|\mu|$ inherits this property? The answer is positive, provided that $E$ is separable, or $E$ is the dual of a separable Banach space, or $E$ has the Radon-Nikodym Property (RNP). In Theorem 2 we consider, in fact, the more general situation of measures with values in $L(E, F)$, where $F$ is another Banach space.

A similar question can be asked for a stochastic function $A: R_{+} \times \Omega \rightarrow E$ with finite variation $|A|$. The variation $|A|$ is measurable, or optional or predictable in case $A$ has this property, or if there is a separable subspace $Z \subset E^{\prime}$ norming for $E$, such that $\langle A, z\rangle$ has this property for every $z \in Z$. In Theorem 3 we consider the more general situation of stochastic functions with values in $L(E, F)$.

Finally, in Theorem 4 we prove that a process $A: R_{+} \times \Omega \rightarrow E$ is measurable, or optional, or predictable, if and only if its continuous part $A^{c}$ and its purely discontinuous part $A^{d}$ have the same property.

2. Stochastic measures with finite variation. Let $\mu: \mathcal{M} \rightarrow E$ be a stochastic measure with finite variation $|\mu|$. We denote by $\mu_{o}$ and $\mu_{p}$ the restriction of $\mu$ to $O$ and $P$, respectively. Then $|\mu|_{o}$ is the restriction of $|\mu|$ to $O$ and we distinguish it from the variation $\left|\mu_{o}\right|$ of $\mu_{o}$ on 0 . Similar considerations for $|\mu|_{p}$ and $\left|\mu_{p}\right|$.

We denote by $\mu^{o}$ and $\mu^{p}$, respectively, the optional and the predictable projection of $\mu$. Then $|\mu|^{o}$ is the optional projection of $|\mu|$ and we distinguish it from the optional extension $\left|\mu_{o}\right|_{e}$ of $\left|\mu_{o}\right|$. Similar considerations for $|\mu|^{p}$ and $\left|\mu_{p}\right|_{e}$. We

Received by the editors April 28, 1987 and, in revised form November 13, 1987.

1980 Mathematics Subject Classification (1985 Revision). Primary 60G07; Secondary 60G70.

Key words and phrases. Stochastic processes, stochastic measures, finite variation, measurable, optional, predictable, Banach space. 
denote by ${ }^{o} \phi$ and ${ }^{p} \phi$, respectively, the optional and the predictable projection of a bounded measurable real process $\phi$.

LEMMA 1. We have

$$
\left|\mu_{o}\right| \leq|\mu|_{o} \quad \text { and } \quad\left|\mu_{p}\right| \leq|\mu|_{p}
$$

If $\mu$ is optional (resp. predictable) then

$$
\left|\mu_{o}\right|=|\mu|_{o} \quad \text { and } \quad|\mu| \leq\left|\mu_{o}\right|_{e}=|\mu|^{o}
$$

$\left(\right.$ resp. $\left|\mu_{p}\right|=|\mu|_{p}$ and $\left.|\mu| \leq\left|\mu_{p}\right|_{e}=|\mu|^{p}\right)$.

ProOF. We consider the optional case only. Let $B \in \mathcal{O}$ and let $\left(B_{i}\right)$ be a finite family of disjoint sets from $O$ contained in $B$. Then

$$
\sum\left\|\mu_{o}\left(B_{i}\right)\right\| \leq \sum|\mu|\left(B_{i}\right)=\sum|\mu|_{o}\left(B_{i}\right) \leq|\mu|_{o}(B)
$$

hence $\left|\mu_{o}\right|(B) \leq|\mu|_{o}(B)$.

Assume now $\mu$ optional and let $A \in \mathcal{M}$; let $\left(A_{i}\right)$ be a finite family of disjoint sets from $\mathcal{M}$ contained in $A$. Then

$$
\begin{aligned}
\sum\left\|\mu\left(A_{i}\right)\right\| & =\sum\left\|\mu\left({ }^{o} 1_{A_{i}}\right)\right\|=\sum\left\|\mu_{o}\left({ }^{o} 1_{A_{i}}\right)\right\| \\
& \leq \sum\left|\mu_{o}\right|\left({ }^{o} 1_{A_{i}}\right)=\sum\left|\mu_{o}\right|_{e}\left(A_{i}\right) \leq\left|\mu_{o}\right|_{e}(A) ;
\end{aligned}
$$

hence $|\mu|(A) \leq\left|\mu_{o}\right|_{e}(A)$. In particular, for $B \in \mathcal{O}$ we have $|\mu|_{o}(B) \leq\left|\mu_{o}\right|(B)$; hence $|\mu|_{o}=\left|\mu_{o}\right|$; consequently $|\mu| \leq\left|\mu_{o}\right|_{e}=|\mu|^{o}$.

REMARK. If $\mu$ and $|\mu|$ are both optional (resp. predictable) then $|\mu|=\left|\mu_{o}\right|_{e}$ (resp. $\left.|\mu|=\left|\mu_{p}\right|_{e}\right)$.

THEOREM 2. Let $\mu: \mathcal{M} \rightarrow L(E, F)$ be an optional (resp. predictable) stochastic measure with finite variation $|\mu|$. Then $|\mu|$ is optional (resp. predictable) in each of the following cases:

(1) $E$ is separable and there is a separable subspace $Z \subset F^{\prime}$ norming for $F$ (in particular this is the case if $E$ and $F$ are separable);

(2) $E$ is separable and $F$ has the RNP;

(3) $\mu$ takes on values in a subspace $G \subset L(E, F)$ having the $R N P$.

PROOF. We consider the optional case only. Let $Z \subset F^{\prime}$ be a subspace norming for $F$. Apply the Radon-Nikodym theorem [2, Theorem 4, p. 263] to the measures $\mu_{o}$ and $\left|\mu_{o}\right|:$ there is a stochastic function $H: R_{+} \times \Omega \rightarrow L\left(E, Z^{\prime}\right)$ such that:

(i) $\|H\| \equiv 1$.

(ii) $\langle H x, z\rangle$ is $\mu_{o}$-measurable for every $x \in E$ and $z \in Z$ and we have $\left\langle\mu_{o}(\phi) x, z\right\rangle$ $=\int\langle H x, z\rangle \phi d\left|\mu_{o}\right|$ for every bounded optional real process $\phi$.

Then for every bounded measurable real process $\phi$ we have

(iii) $\langle\mu(\phi) x, z\rangle=\int\langle H x, z\rangle \phi d\left|\mu_{o}\right|_{e}$.

In fact, for each $x \in E$ and $z \in Z$ choose $H_{x, z}: R_{+} \times \Omega \rightarrow R$ optional such that

$$
H_{x, z}=\langle H x, z\rangle, \quad\left|\mu_{o}\right| \text {-a.e. }
$$


Then

$$
\begin{aligned}
\langle\mu(\phi) x, z\rangle & =\left\langle\mu\left({ }^{o} \phi\right) x, z\right\rangle=\int\langle H x, z\rangle^{o} \phi d\left|\mu_{o}\right| \\
& =\int H_{x, z}{ }^{o} \phi d\left|\mu_{o}\right|=\int{ }^{o}\left(H_{x, z} \phi\right) d\left|\mu_{o}\right| \\
& =\int\left(H_{x, z} \phi\right) d\left|\mu_{o}\right|_{e}=\int\langle H x, z\rangle \phi d\left|\mu_{o}\right|_{e}
\end{aligned}
$$

If $E$ and $Z$ are separable, we deduce from [2, Theorem 6, p. 274] that $|\mu|=$ $\|H\|\left|\mu_{o}\right|_{e}=\left|\mu_{o}\right|_{e}$ hence $|\mu|$ is optional, and this proves case (1).

Assume now that $E$ is separable and $F$ has the RNP and prove that there is a separable subspace $F_{0} \subset F$ such that $\mu$ takes on values in $L\left(E, F_{0}\right)$; we can then apply case (1) to deduce that $|\mu|=\left|\mu_{o}\right|_{e}$. For this purpose let $x \in E$ and define $\nu: \mathcal{M} \rightarrow F$ by $\nu(M)=\mu(M) x$ for $M \in \mathcal{M}$. Then $|\nu| \leq|\mu|\|x\|$. Since $F$ has the RNP there is a function $H_{x} \in L_{F}^{1}(|\mu|)$ such that

$$
\nu(M)=\int_{M} H_{x} d|\mu| \text { for } M \in \mathcal{M} .
$$

We can choose $H_{x}$ separably valued and then $\nu$ takes on values in the separable subspace $F_{x} \subset F$ generated by the range of $H_{x}$. If $E_{0}$ is a countable dense subset of $E$, the space $F_{0}$ generated by the family $\left\{F_{x} ; x \in E_{0}\right\}$ is separable and for every $x \in E$ and $M \in \mathcal{M}$ we have $\mu(M) x \in F_{0}$, that is, $\mu$ takes on values in $L\left(E, F_{0}\right)$. Case (3) reduces to case (2) by considering $G=L(R, G)$.

REMARKS. $1^{\circ}$. If $\mu: \mathcal{M} \rightarrow E$ is an optional (resp. predictable) stochastic measure with finite variation, then its variation $|\mu|$ is optional (resp. predictable) in each of the following cases:

(a) $E$ is the dual of a spearable Banach space;

(b) $E$ is separable;

(c) $E$ has the RNP.

In fact, we apply the preceding theorem for $E=L(R, E)$.

$2^{\circ}$. A real stochastic measure $\mu$ is optional (resp. predictable) iff $\mu^{+}$and $\mu^{-}$ have this property.

3. Stochastic functions with finite variation. Let $A: R_{+} \times \Omega \rightarrow E$ be a stochastic function with finite variation $|A|_{[a, b]}$ on each compact interval $[a, b]$. For each $t \geq 0$ we set

$$
|A|_{t}=\left\|A_{0}\right\|+|A|_{[0, t]} .
$$

The stochastic function $|A|=\left(|A|_{t}\right)_{t \geq 0}$ is called the variation of $A$. For $s<t$ we have

$$
\left\|A_{t}-A_{s}\right\| \leq|A|_{t}-|A|_{s}=|A|_{[s, t]}
$$

which proves that $A$ has left and right limits at any point. From Proposition 5 below we deduce that $A$ is right or left continuous if and only if $|A|$ has the same property.

Assume that $A$ is right continuous. We can decompose

$$
A=A^{c}+A^{d},
$$


where $A^{c}$ is continuous and $A^{d}$ is purely discontinuous, and we have

$$
A_{t}^{d}=\sum_{s \leq t} \Delta A_{s}
$$

where $\Delta A_{s}=A_{s}-A_{s-}$ is the jump of $A$ at $s$. We then have (see Proposition 7 below)

$$
|A|^{c}=\left|A^{c}\right| \text { and }|A|^{d}=\left|A^{d}\right| .
$$

If $u \in L(E, F)$ then we have also

$$
(u A)^{c}=u A^{c} \text { and }(u A)^{d}=u A^{d} .
$$

In particular, if $A: R_{+} \times \Omega \rightarrow L(E, F)$, then for every $x \in E$ and $z \in F^{\prime}$ we have

$$
\begin{aligned}
(A x)^{c} & =A^{c} x \quad \text { and }(A x)^{d}=A^{d} x, \\
\langle A x, z\rangle^{c} & =\left\langle A^{c} x, z\right\rangle \text { and }\langle A x, z\rangle^{d}=\left\langle A^{d} x, z\right\rangle .
\end{aligned}
$$

THEOREM 3. Let $A: R_{+} \times \Omega \rightarrow E$ be a right continuous stochastic function with finite variation $|A|$. Then $A$ is measurable (resp. optional, predictable) iff $A^{c}$ and $A^{d}$ have this property.

ProOf. Assume $A$ is measurable (resp. optional, predictable) and prove that $A^{d}$ has the same property. Then, by difference, $A^{c}$ will have the same property.

The set $U=\left\{(t, \omega) ; A_{t}(\omega) \neq A_{t-}(\omega)\right\}$ is measurable (resp. optional, predictable). Theorem IV, $88 \mathrm{~B}$ in [1] remains valid for vector-valued processes: $U$ is a countable union of disjoint graphs $\left[S_{n}\right]$ of positive random variables (resp. optional stopping times, predictable stopping times). For each $n, \Delta A_{S_{n}} I_{\left\{S_{n}<\infty\right\}}$ is $\mathcal{F}$-measurable

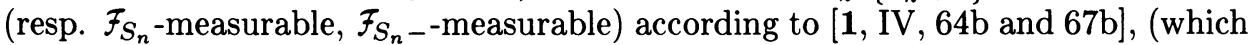
remain valid for vector-valued processes), and

$$
A_{t}^{d}=\sum_{1 \leq n<\infty} \Delta A_{S_{n}} I_{\left\{S_{n} \leq t\right\}} .
$$

Assume first that $A$ is optional. Then $\Delta A_{S_{n}} I_{\left[S_{n}, \infty[\right.}$ is adapted and right continuous, therefore $A^{d}$ is optional.

If $A$ is measurable, it is optional with respect to the constant filtration $\mathcal{F}_{t}=\mathcal{F}$; by the above, $A^{d}$ is measurable.

Assume now that $A$ is predictable. To prove that $A^{d}$ is predictable we apply, to each process $Y=\Delta A_{S_{n}} I_{\left[S_{n}, \infty\right.}$, Theorem IV, 88C in [1] (which remains valid for vector-valued processes). If $T$ is a totally inaccessible stopping time then

$$
Y_{T}-Y_{T-}=\Delta A_{S_{n}} I_{\left\{S_{n}=T<\infty\right\}}
$$

By $[1, \mathrm{IV}, 81 \mathrm{~b}]$, we have $P\left\{S_{n}=T<\infty\right\}=0$, since $S_{n}$ are predictable; therefore $Y_{T}=Y_{T-}$ a.s. and condition 1) of Theorem IV, $88 \mathrm{C}$ in [1] is satisfied. If $T$ is any stopping time (not necessarily predictable), then

$$
Y_{T} I_{\{T<\infty\}}=\Delta A_{S_{n}} I_{\left\{S_{n}<\infty\right\}} I_{\left\{S_{n} \leq T\right\}}
$$

We mentioned above that $\Delta A_{S_{n}} I_{\left\{S_{n}<\infty\right\}}$ is $\mathcal{F}_{S_{n}}$-measurable. Then by $[\mathbf{1}, \mathrm{IV}, 73 \mathrm{~b}]$, $Y_{T} I_{\{T<\infty\}}$ is $\mathcal{F}_{T}$-measurable and condition 2) of Theorem IV, 88C in [1] is also satisfied. Therefore, $Y$ is predictable; consequently $A^{d}$ is also predictable. 
THEOREM 4. Let $A: R_{+} \times \Omega \rightarrow L(E, F)$ be a right continuous stochastic function with finite variation $|A|$. In each of the following cases, $|A|,|A|^{d}$ and $|A|^{c}$ are measurable (resp. optional, predictable) :

(1) $A$ is measurable (resp. optional, predictable);

(2) $E$ is separable and for every $x \in E, A x$ is measurable (resp. optional, predictable);

(3) $E$ is separable and there exists a separable subspace $Z \subset F^{\prime}$ norming for $F$, such that for every $x \in E$ and $z \in Z,\langle A x, x\rangle$ is measurable (resp. optional, predictable).

ProOF. For every natural $n$ and every $t \geq 0$ set

$$
|A|_{t}^{n}=\left\|A_{0}\right\|+\sum_{1 \leq k<\infty}\left\|A_{(k+1) 2^{-n} \wedge t}-A_{k 2^{-n} \wedge t}\right\| .
$$

By right continuity of $A$ we have

$$
|A|=\sup _{n}|A|^{n}
$$

In each of the cases (1)-(3), the stochastic function $\left(\left\|A_{a \wedge t}-A_{b \wedge t}\right\|\right)_{t \geq 0}$ is measurable (resp. optional, predictable) for any $a \geq 0$ and $b \geq 0$. It follows that each $|A|^{n}$ is measurable (resp. optional, predictable), hence $|A|$ has the same property. The assertion about $|A|^{c}$ and $|A|^{d}$ is obtained by applying the preceding theorem to $|A|$.

REMARK. As a particular case, considering $E=L(R, E)$, we deduce that if $A: R_{+} \times \Omega \rightarrow E$ is a right continuous function with finite variation $|A|$, then $|A|,|A|^{c}$ and $|A|^{d}$ are measurable (resp. optional, predictable) in each of the following cases:

(1) $A$ is measurable (resp. optional, predictable);

(2) $A$ is weakly measurable (resp. weakly optional, weakly predictable) and there is a separable subspace $Z \subset E^{\prime}$ norming for $E$;

(3) $E$ is the dual of a separable Banach space $G$ and $A$ is weak*-measurable (resp. weak*-optional, weak* ${ }^{*}$-predictable).

A theorem similar to the preceding one can be obtained (for the measurable and optional cases only) without separability assumptions on the Banach spaces involved, but imposing a "lifting condition" on $A$.

THEOREM 5. Let $A: R_{+} \times \Omega \rightarrow L(E, F)$ be a right continuous stochastic function with finite variation $|A|$. Assume there is a subspace $Z \subset F^{\prime}$ norming for $F$ such that $\langle A x, z\rangle$ is measurable (resp. optional) for every $x \in E$ and $z \in Z$. Assume further that there exists a lifting $\rho$ of $P$ such that $\rho\left[A_{t}\right]=A_{t}\left(\right.$ resp. $\left.\rho\left(A_{t}\right)=A_{t}\right)$ for $t \geq 0$ (see [2, p. 212]). Then $|A|,|A|^{d}$ and $|A|^{c}$ are measurable (resp. optional).

PROOF. If $\rho\left[A_{t}\right]=A_{t}$ for every $t \geq 0$, then $\rho\left[A_{a \wedge t}-A_{b \wedge t}\right]=A_{a \wedge t}-A_{b \wedge t}$ hence $\left\|A_{a \wedge t}-A_{b \wedge t}\right\|$ is $\mathcal{F}$-measurable for every $a \geq 0$ and $b \geq 0$ (see [3, Proposition 5, p. 213]). By right continuity, the process $\left(\left\|A_{a \wedge t}-A_{b \wedge t}\right\|\right)_{t \geq 0}$ is measurable and we deduce that $|A|,|A|^{c}$ and $|A|^{d}$ are measurable as in the proof of Theorem 4 .

Assume now that $\langle A x, z\rangle$ is optional for $x \in E, z \in Z$, and that $\rho\left(A_{t}\right)=A_{t}$ for $t \geq 0$; that is, $\rho\left(\left\langle A_{t} x, z\right\rangle\right)=\left\langle A_{t} x, z\right\rangle$ for $x \in E$ and $z \in Z$. The restriction $\rho_{t}$ of $\rho$ to $L^{\infty}\left(\mathcal{F}_{t}, P\right)$ is a lifting of $L^{\infty}\left(\mathcal{F}_{t}, P\right)$ and we have $\rho_{t}\left(A_{s}\right)=A_{s}$ for $s \leq t$; in particular, $\rho_{t}\left(A_{a \wedge t}-A_{b \wedge t}\right)=A_{a \wedge t}-A_{b \wedge t}$, hence $\left\|A_{a \wedge t}-A_{b \wedge t}\right\|$ is $\mathcal{F}_{t}$-measurable, consequently the process $\left(\left\|A_{a \wedge t}-A_{b \wedge t}\right\|\right)_{t \geq 0}$ is optional; then $|A|,|A|^{c}$ and $|A|^{d}$ are optional, as in the proof of Theorem 4. 
4. Appendix. In this section we prove some properties of functions with finite variation, which were used in this paper and in other papers.

PROPOSITION 6. Let $f: R_{+} \rightarrow E$ be a function with finite variation $|f|$. Then $f$ is right or left continuous iff $|f|$ has the same property.

ProOF. As we noted before, if $|f|$ is right (or left) continuous, then the right (or left) continuity of $f$ follows from the inequality:

$$
|f(t)-f(s)| \leq|f|(t)-|f|(s) \text { for } s<t .
$$

Conversely assume that $f$ is right continuous at a point $a \in R_{+}$but $M=|f|(a+)-$ $|f|(a)>0$ and prove that we reach a contradiction. Let $b>a$ and $\varepsilon>0$. We can find by induction a strictly decreasing sequence $\left(x_{n}\right)$ in $R_{+}$with $x_{1}=b$ and $x_{n} \rightarrow a$, and for each $n$, a partition $\left(d_{n}\right)$ of $\left[x_{n+1}, x_{n}\right]$ such that

$$
V_{d_{n}}:=\sum_{t_{i} \in d_{n}}\left\|f\left(t_{i+1}\right)-f\left(t_{i}\right)\right\| \geq M-\varepsilon 2^{-n} .
$$

In fact, let $\Delta_{1}$ be a partition of $[a, b]$ such that

$$
V_{\Delta_{1}}>|f|_{[a, b]}-\varepsilon / 4 \geq M-\varepsilon / 4 \text {. }
$$

Since $f$ is right continuous at $a$, there is $x_{2}>a$ such that if $x \in\left[a, x_{2}\right]$, then $\|f(x)-f(a)\|<\varepsilon / 4$. We can choose $x_{2} \leq \inf \left\{t \in \Delta_{1}, t>a\right\}$ and add $x_{2}$ to $\Delta_{1}$. Denote $d_{1}=\Delta_{1}-\{a\}$. Then $d_{1}$ is a partition of $\left[x_{2}, x_{1}\right]$ and

$$
V_{d_{1}}=V_{\Delta_{1}}-\left\|f\left(x_{2}\right)-f(a)\right\| \geq M-\varepsilon / 4-\varepsilon / 4=M-\varepsilon 2^{-1} \text {. }
$$

The second step of the induction process is proved similarly. For every $n$ we then have

$$
|f|_{[a, b]} \geq \sum_{1 \leq i \leq n}|f|_{\left[x_{i+1}, x_{i}\right]} \geq \sum_{1 \leq i \leq n} V_{d_{i}} \geq n M-\varepsilon,
$$

hence $|f|_{[a, b]}=\infty$, which contradicts the hypothesis.

PROPOSITION 7. Let $f: R_{+} \rightarrow E$ be a right continuous function with finite variation $|f|$. Then:

(a) $|f|=\left|f^{c}\right|+\left|f^{d}\right|$

(b) $|f|^{c}=\left|f^{c}\right|$ and $|f|^{d}=\left|f^{d}\right|$;

(c) $\Delta|f|=\|\Delta f\|$.

PROOF. Let $\mu_{f}$ be the Stieltjes measure on $R_{+}$corresponding to $f$ :

$$
\mu_{f}(\{0\})=\|f(0)\|, \quad \mu_{f}((a, b])=f(b)-f(a) .
$$

Then [2, p. 362] $\left|\mu_{f}\right|=\mu_{|f|}$. The measure $\mu_{f^{d}}$ is discrete (atomic) and the measure $\mu_{f^{c}}$ is diffuse (nonatomic) and they are supported by disjoint sets; therefore

$$
\left|\mu_{f}\right|=\left|\mu_{f^{c}}+\mu_{f^{d}}\right|=\left|\mu_{f^{c}}\right|+\left|\mu_{f^{d}}\right| \text {. }
$$

On the other hand

$$
\left|\mu_{f}\right|=\mu_{|f|}=\mu_{|f|^{c}}+\mu_{|f|^{d}} .
$$

From the uniqueness of the decomposition we deduce

$$
\mu_{|f|^{c}}=\mu_{\left|f^{c}\right|} \text { and } \mu_{|f|^{d}}=\mu_{\left|f^{d}\right|}
$$

therefore, $|f|^{c}=\left|f^{c}\right|$ and $|f|^{d}=\left|f^{d}\right|$, and $|f|=\left|f^{c}\right|+\left|f^{d}\right|$. In particular, for any $a \in R_{+}$we have

$$
\Delta|f|(a)=\mu_{|f|}(\{a\})=\left|\mu_{f}\right|(\{a\})=\left\|\mu_{f}(\{a\})\right\|=\|\Delta f(a)\| .
$$




\section{BIBLIOGRAPHY}

1. C. Dellacherie and P. Meyer, Probabilities and potentials, Vols. I, II, North-Holland, 1978, 1980.

2. N. Dinculeanu, Vector measures, Pergamon Press, Oxford, 1967.

3. _ Vector-valued stochastic processes. I. Vector measures and vector-valued stochastic processes with finite variation, J. Theoret. Probab. 1 (1988), 149-169.

4. __ Vector-valued stochastic processes. III. Projections and dual projections, Seminar on Stochastic Processes, 1987, Birkhauser, 1988.

5. A. and C. Ionescu Tulcea, Topics in the theory of lifting, Springer-Verlag, Berlin, Heidelberg and New York, 1969.

6. M. Métivier, Semimartingales, De Gruyter, Berlin, 1982.

Department of Mathematics, University of Florida, Gainesville, Florida 32611 